\title{
Impact of Age on Cognitive and Psychological Functioning in Patients with Diabetes
}

\author{
Halder $\mathbf{S}^{*}$, Samajdar $\mathrm{S}^{2}$ and Mahato $\mathrm{AK}^{1}$ \\ ${ }^{1}$ Associate Professor, Amity University, Kolkata, India \\ ${ }^{2}$ Clinical Psychologist, Kolkata, India
}

"Correspondence: Susmita Halder, Associate Professor, Amity University, Kolkata, India

Received on 27 January 2021; Accepted on 19 March 2021; Published on 24 March 2021

Copyright (C) 2021 Halder S, et al. This is an open access article and is distributed under the Creative Commons Attribution License, which permits unrestricted use, distribution, and reproduction in any medium, provided the original work is properly cited.

\begin{abstract}
Diabetes mellitus is a chronic and non-communicable disease which is also termed as "sugar" emerged as one of the leading causes of global health deterioration. It is associated with decreased physiological and psychological functioning. Age is a significant predictor to evolve diabetes mellitus. Cognitive changes due to diabetes and psychosocial distress are prominent among the population and the risk is high with increasing age range. The aim of the present study is to find out any association between age and cognitive and psychological functioning of patients with diabetes. In the present study, age range of the sample was selected as 40-60 years. A total of 60 individuals of both genders were selected; 30 individuals with diagnosis of diabetes mellitus and equal number of normal controls were included. The cognitive functioning was measured by neuropsychological tools and the psychological functioning was measured by Psychological General Well-being Index. The results indicate that in case of diabetes patients, age was found to be mostly significant and negatively correlated with cognitive and psychological functioning in comparison to normal controls. Findings from the present study suggest that age could be a predictor in changed cognitive and psychological functioning of patients with diabetes and increased age showed poorer cognitive and psychological functioning.
\end{abstract}

Keywords: diabetes mellitus, ageing, cognitive functioning, psychological functioning

\section{Introduction}

In public health significance, ageing population is being rapidly prevalent to diabetes mellitus (DM) which is a chronic metabolic disease condition and is associated with time-related multimodal complications. Complications might have physiological and psychological negative impact. The prevalence of diabetes and glucose intolerance increases with aging [1]. With the anticipated further aging, the burden of diabetes and impaired glucose tolerance (IGT) is increasing 
in the population. Aging is a major contributing factor to the diabetes epidemic, and aged individuals are the representative of the fastest growing parts of the population of diabetes. Age-related insulin resistance is interlinked with negative impact in the body composition and physical inactivity among the population [2] which might have explained the benefits of the intensive lifestyle intervention and modification in aging. Previous studies have reported cognitive decline in both type 1 and type 2 patients with diabetes and decreased mental efficiency has been repeatedly reported. Influence of the metabolic syndrome in the aging population involves deficit in memory and learning, problem solving, and mental and motor speed [3, 4].

The risk factors of cognitive dysfunction in diabetes mellitus might have influenced the glycemic control, inflammation, hypoglycemia, and psychological distress [5]. The progressive impact of these conditions on the vascular disorders decreased the threshold and the cognitive functioning is affected in the aging brain of diabetic patients. Though chronic diseases, including diabetes self-care, involve much health-promoting behavior that is required for various degrees of cognitive pliability, insight, and knowledge to function proper self-care coordination and planning. Monitoring glucose levels, regular medications, insulin injections, dietary managements, lifestyle modifications and exercise require active participation of patients and it can have impact upon different domains of cognitive functioning. In addition, the recognition of disease condition, treatment procedure, and hypoglycemia prevention, are critical for the older population. Combined with rapid population aging, the health system in India needs to prepare to care for large number of old diabetic patients with an overabundance of prolonged complications; both vascular and metabolic. Different reports of psychological illness in younger patients with diabetes are taken into account in literature but consideration of aging population is rare. The critical effect of chronic diabetes on physiological well-being has been documented along with its socioeconomic connotations. Older patients are more at risk to chronic diabetic unfavorable complications due to prolonged exposure to negative metabolic and vascular impacts. The vulnerability to hypoglycemia and its adverse outcome has been well identified, necessitating immediate glycemic control. However, the decreased mental health status of older diabetic patients has received inadequate medical attention in mental health practice.

The aim of the present study is to correlate increasing age range and patients with diabetes in cognitive and psychological functioning.

\section{Materials and Methods}

\section{Participants}

In the present study, 60 individuals of both genders were selected and divided into two equal groups of 30 who were diagnosed with diabetes mellitus and normal controls. Inclusion criteria of the individuals with diabetes was 1) diagnosed with diabetes, 2) duration of illness is minimum 5 years, 3) age range of 40-60 years, 4) both genders 5) minimum education level was eighth grade. Patients with other medical/psychological/neurological disorders, were excluded from the sample. In case of normal controls, the inclusion criteria was 1) not diagnosed with diabetes, 2) age range of 40-60 years, 3) both genders, 4) minimum educational qualification was eighth grade. Any other medical, neurological and psychiatric conditions were excluded from this group. All the data was collected from the participants and consent form was provided to them.

\section{Measures}

- Socio-demographic and clinical datasheet: A tailor-made, semi-structured, socio-demographic and clinical datasheet was provided to the participants to obtain the information regarding demographic details, duration of illness, comorbid conditions, etc.

- Verbal working memory n-back test: The verbal working memory 1 back and 2 back tests were used to assess the verbal working memory. It requires verbal storage, rehearsal and information manipulation. The Indian norms for the test were used in the present study [6]. 
- Digit symbol substitution test (DSST): DSST was used to assess the coordination of visuo-motor functioning, motor persistence, sustained attention, and processing speed of the participants. The Indian norms for the test were used in the present study [6].

- Trail making test (TMT): TMT measured the visual attention and task switching. It assesses the sustained attention, information processing including visual search speed, scanning, processing speed, cognitive flexibility, and executive functioning [7].

- Stroop neuropsychological screening test: Stroop test assesses the perceptual set-shifting ability and it can be shifted on demand, suppressing a habitual response in favor of an unusual one [8].

- Psychological General Well-being Index: The Psychological General Well-being Index is an assessment tool of the level of subjective psychological well-being. It assesses subjective representations of sense of subjective well-being and it consists of 22 standardized items. The tool produces a single measure of psychological well-being under 6 subscale domains, namely, anxiety, depression, positive well-being, selfcontrol, general health, and vitality. Higher scores on the scale indicate a greater sense of well-being [9].

\section{Statistical analysis}

In the present study, after the scales were scored, quantitative analysis was conducted by descriptive statistics. Pearson $\mathrm{r}$ correlation was also done to find the relationship between variables.

\section{Results}

The following tables (Table 1,2 and 3) show the details of sample and the relationship between age and cognitive and psychological variables in diabetes patients.

\begin{tabular}{|c|c|c|c|c|c|}
\hline \multirow{2}{*}{\multicolumn{2}{|c|}{ Variables }} & \multirow{2}{*}{\multicolumn{2}{|c|}{$\begin{array}{l}\text { Patients with diabetes } \\
(\text { Mean } \pm \text { SD })\end{array}$}} & \multirow{2}{*}{\multicolumn{2}{|c|}{$\begin{array}{l}\text { Normal controls } \\
(\text { Mean } \pm \text { SD })\end{array}$}} \\
\hline & & & & & \\
\hline \multicolumn{2}{|l|}{ Age } & \multicolumn{2}{|c|}{$52.72(7.19)$} & \multicolumn{2}{|c|}{$51.25(6.89)$} \\
\hline & & Number & Percentage & Number & Percentage \\
\hline \multirow{2}{*}{ Sex } & Male & 15 & 50.00 & 16 & 53.33 \\
\hline & Female & 15 & 50.00 & 14 & 46.67 \\
\hline \multirow{3}{*}{ Educational qualification } & $10+2$ & 8 & 26.67 & 7 & 23.33 \\
\hline & Graduate & 20 & 66.67 & 15 & 50.00 \\
\hline & Post-graduate & 12 & 40.00 & 8 & 26.67 \\
\hline \multirow[t]{3}{*}{ Occupation } & Service & 18 & 60.00 & 20 & 66.67 \\
\hline & Business & 5 & 16.67 & 5 & 16.67 \\
\hline & Homemaker & 7 & 23.33 & 5 & 16.67 \\
\hline \multirow{3}{*}{ Socioeconomic status } & Lower middle-class & 5 & 16.67 & 3 & 10.00 \\
\hline & Middle-class & 9 & 30.00 & 10 & 33.33 \\
\hline & Upper middle-class & 16 & 53.33 & 7 & 23.33 \\
\hline \multirow{3}{*}{ Marital status } & Married & 22 & 73.33 & 19 & 63.33 \\
\hline & Unmarried & 5 & 16.67 & 10 & 33.33 \\
\hline & Divorced & 3 & 10.00 & 1 & 03.33 \\
\hline
\end{tabular}

Table 1: Socio-demographic details of the sample. 


\begin{tabular}{|c|c|c|c|c|}
\hline Cognitive variables & & & Patients with diabetes & Normal controls \\
\hline & & & \multicolumn{2}{|c|}{$\mathrm{r}$ value in relation with age } \\
\hline Cognitive flexibility & Stroop effect & & $-0.400^{*}$ & 0.050 \\
\hline \multirow{6}{*}{ Working memory } & \multirow{3}{*}{ n-back 1} & Hit & 0.054 & 0.349 \\
\hline & & Omission & 0.010 & -0.349 \\
\hline & & Commission & 0.051 & 0.192 \\
\hline & \multirow{3}{*}{ n-back 2} & Hit & $-0.447^{*}$ & 0.053 \\
\hline & & Omission & $0.447^{*}$ & -0.095 \\
\hline & & Commission & -0.045 & 0.013 \\
\hline \multirow{2}{*}{ Sustained attention } & \multicolumn{2}{|c|}{ TMT-1 (Total time) } & $0.414^{*}$ & 0.197 \\
\hline & \multicolumn{2}{|c|}{ TMT-2 (Total time) } & $0.524^{* * *}$ & -0.078 \\
\hline \multirow{2}{*}{ Processing speed } & \multirow{2}{*}{ DSST } & Time & $0.472^{* *}$ & $0.390^{*}$ \\
\hline & & Errors & 0.265 & 0.262 \\
\hline
\end{tabular}

Table 2: Relationship between age and cognitive functioning of patients with diabetes and normal controls.

\begin{tabular}{|l|l|l|}
\hline Psychological variables & \multicolumn{1}{|c|}{ Patients with diabetes } & Normal controls \\
\hline & \multicolumn{2}{|c|}{ r value in relation with age } \\
\hline Positive well-being & $-0.022^{*}$ & -0.089 \\
\hline Self-control & $-0.163^{*}$ & 0.129 \\
\hline Anxiety & 0.227 & -0.040 \\
\hline Depression & -0.040 & 0.012 \\
\hline Vitality & $0.152^{*}$ & -0.128 \\
\hline General health & $-0.330^{*}$ & 0.036 \\
\hline
\end{tabular}

Table 3: Relationship between age and psychological functioning of patients with diabetes and normal controls.

\section{Discussion}

The present study was aimed to find out any relationship between increasing age and cognitive and psychological functioning in patients with diabetes. Overall results suggested that, there is a significant relationship between age, cognitive and psychological functioning in the sample variables (Table 2 and 3). For further analysis of sample and results, discussion was made following the tables presented in the results section. The table showed the sociodemographic details of the sample which indicated that the mean age of the sample has been found to be 52 and equal number of male and female participants were present in the sample (Table 1). The mean age in the current study is going with the trend of prevalence age of diabetes mellitus which is 50-55 years and the probability of diagnosis of diabetes is higher among older age [2]. Most of the individuals who participated in the study were graduate and belong to upper middle-class socioeconomic background. Majority of the sample were working professionals and most of them were married. It has been found that the average duration of illness has been found to be 11 years (approximately).

Cognitive and psychological domains were chosen for this study as diabetes mellitus is a chronic illness and it may have a long-time impact on the patients' physiological, cognitive and psychological wellbeing. Patients with chronic diabetes need special medical attention and intervention for psychological needs [10]. In cognitive domains, the table showed that there is a significant relationship between increased age and overall cognitive functioning (Table 2). Further analysis suggests that there is a significant negative relationship between cognitive flexibility and ageing in patients with diabetes mellitus. Specifically, the Stroop task indicates the ability to response inhibition and cognitive flexibility. Following the scores in the present study, it's indicative that the increased age range is associated with declined response inhibition and cognitive flexibility in diabetes mellitus. Significant changes in the domain of response inhibition might have an adverse manifestation of diabetes-associated cognitive dysfunction across different age groups $[11,12]$. In the domain of working memory, sustained attention and processing speed, there is a significant relationship with the ageing of patients with diabetes, suggesting more age is leading to poor performance in these domains. In comparison to normal controls, the patients with diabetes, glycosylated hemoglobin (HbA1c) is a marker of glycemic control and glucose intolerance, and $\mathrm{HbA1c}$ of more than $7 \%$ was interrelated with an increase in 
occurrence of mild cognitive impairment [13]. Deficits in working memory, frontal lobe executive functioning, learning and complex psychomotor process and abilities have also been found associated with a higher level of HbA1c with ageing. So, it can be said that impaired glucose tolerance in association with diagnosis of diabetes has been associated with cognitive alteration $[14,15]$. Diabetes related hypoglycemic episodes are related to rapid changes in central nervous system and insulin resistance may have a more important role than an elevated fasting glucose level in increasing the risk for mild cognitive impairment in aged patients with diabetes. Difference between normal cognitive ageing and diabetes related cognitive changes can be significantly distinguishing. Impaired glucose tolerance mostly affects the younger brain and along with increasing age it becomes prominent. Significance of structural and functional changes which includes increased neuron loss, decreased synaptic density, declined energy production, disordered synaptic signaling and destructed protein homeostasis due to hypoglycemia with increasing age and thus, cognitive functioning declines much faster among individuals with diabetes.

In the domain of psychological functioning, the table indicates the Psychological General Well-being Index in terms of subjective feeling of well-being related to psychological functioning of the patients (Table 3). Results in present study showed that, increased age has significant association with negative perception of well-being, self-control and health conditions. Patients with diabetes have comorbid psychological distress due to prolong nature of the disease condition which might have impact on interpersonal relationships, occupational functioning, and quality of life. Through aging, gradual decrease in functional abilities [16] can be characterized and it is a process of declination of physiological and psychological deformities. Different chronic disease conditions which are associated with diabetes are mainly associated with elderly population [17]. In comparison to normal controls, diabetic condition is facing unfavorable and undesirable changes in life which might have implications on control over health status and leading to adverse physiological complications [18]. Patients with diabetes have critical perception related to their own health and consequence due to their age-related risk factors and because of their declined physical functions [19]. They might have developed anxious coping styles i.e., avoidance of critical situations or escape and denial. Perception of self could have an impact on self-care regimens, lifestyle modifications and in turn that can increase diabetic complications. With increasing age of diabetes individuals, requirements of self-care, daily living chores and barriers in regard with psychosocial functioning take a huge part of changing lifestyle. Impact of chronic illness itself has an implication to alternate quality of life of patients. In the present study, it has been seen that, the perception of wellbeing and general health is becoming poor with increased age, though with increasing age, acceptance of chronic condition and being resilient with the changing lifestyle is more prominent among the present sample.

\section{Conclusion}

The degree, pattern, and nature of the quality of life in terms of cognitive and psychological functioning are eventually decreasing with age though in case of individuals with diabetes the decline is more prominent and irreversible as well. Diabetes-related distress has implications upon perception of general health and psychological well-being. Increased age has a significant role in changes in overall functioning of the individuals with diabetes mellitus.

\section{References}

1. Chang AM, Halter JB. Aging and insulin secretion. Am J Physiol Endocrinol Metab. 2003;284(1):E7-12.

2. Kalyani RR, Golden, SH, Cefalu WT. Diabetes and aging: Unique considerations and goals of care. Diabetes Care. 2017;40(4);440-43.

3. Enzinger C, Fazekas F, Matthews PM, et al. Risk factors for progression of brain atrophy in aging: six-year follow-up of normal subjects. Neurology. 2005;64(10):1704-711.

4. Ryan CM. Neurobehavioral complications of type I diabetes. Examination of possible risk factors. Diabetes Care. 1988;11(1):86-93. 
5. Feinkohl I, Price JF, Strachan MW, et al. The impact of diabetes on cognitive decline: potential vascular, metabolic, and psychosocial risk factors. Alzheimers Res Ther. 2015;7(1):46.

6. Rao SL, Subbakrishna DK, Gopukumar K. NIMHANS Neuropsychological Battery-2004, Manual. Bangalore, India: National Institute of Mental Health and Neurosciences; 2004.

7. Lezak MD. Trail making test. In: Neuropsychological Assessment. 3rd ed. New York, NY: Oxford University Press; 1995.

8. Trenerry MR, Crosson B, DeBoe J, et al. Stroop Neuropsychological Screening Test Manual. Lutz FL: Psychological Assessment Resources; 1989.

9. Dupuy HJ. The Psychological General Well-Being Index (PGWB). 1971.

10. Kalra S, Sridhar GR, Balhara YP, et al. National recommendations: Psychosocial management of diabetes in India. Indian J Endocrinol Metab. 2013;17(3):376-95.

11. Manschot SM, Brands AM, Grond van der J, et al. Brain magnetic resonance imaging correlates of impaired cognition in patients with type 2 diabetes. Diabetes. 2006;55(4):1106-113.

12. Yeung SE, Fischer AL, Dixon RA. Exploring effects of type 2 diabetes on cognitive functioning in older adults. Neurospychology. 2009;23(1):1-9.

13. Yaffe K, Blackwell T, Whitmer RA, et al. Glycosylated hemoglobin level and development of mild cognitive impairment or dementia in older women. J Nutr Health Aging. 2006;10(4):293-95.

14. Halder S, Samajdar S, Mahato AK. An exploratory study of cognitive functioning and psychological wellbeing in middle-aged adults with diabetes mellitus. J Social Health Diab.2020;8(01):008-012.

15. Kanaya AM, Barrett-Connor E, Gildengorin G, et al. Change in cognitive function by glucose tolerance status in older adults: A 4-year prospective study of the Rancho Bernardo study cohort. Arch Intern Med. 2004;164(12):1327-333.

16. Tessier D, Meneilly GS. Diabetes management in the elderly. In: Gerstein HC, Haynes RB, eds. Evidencebased diabetes care. Hamilton: BC Decker, 2001: pg. 370-79.

17. Roy T, Lloyd CE. Epidemiology of depression and diabetes: A systematic review. J Affect Disord. 2012;142(Suppl.):S8-21.

18. Pouwer F. Should we screen for emotional distress in type 2 diabetes mellitus? Nat Rev Endocrinol. 2009;5(12):665-71.

19. Peyrot M, McMurry JF Jr., Kruger DF. A biopsychosocial model of glycemic control in diabetes: Stress, coping and regimen adherence. J Health Soc Behav. 1999;40(2):141-58. 